\title{
Navigation for Fluoroscopy-Guided Cryo-Balloon Ablation Procedures of Atrial Fibrillation
}

\author{
Felix Bourier ${ }^{a}$, Alexander Brost ${ }^{b}$, Andreas Kleinoeder ${ }^{b}$, Tanja \\ Kurzendorfer $^{b}$, Martin Koch ${ }^{b}$, Atilla Kiraly ${ }^{c}$, Hans-Juergen Schneider ${ }^{a}$, \\ Joachim Hornegger $^{b, d}$, Norbert Strobel ${ }^{e}$, and Klaus Kurzidim ${ }^{a}$, \\ ${ }^{a}$ Klinik für Herzrhythmusstörungen, Krankenhaus Barmherzige Brüder, \\ Regensburg, Germany \\ ${ }^{b}$ Pattern Recognition Lab, Friedrich-Alexander-University \\ Erlangen-Nuremberg, Erlangen, Germany \\ ${ }^{c}$ Siemens Corporate Research, Princeton, NJ, USA \\ ${ }^{d}$ School in Advanced Optical Technologies (SAOT), Erlangen, Germany \\ ${ }^{e}$ Siemens AG, Healthcare Sector, Forchheim, Germany
}

\begin{abstract}
Atrial fibrillation (AF), the most common arrhythmia, has been identified as a major cause of stroke. The current standard in interventional treatment of AF is the pulmonary vein isolation (PVI). PVI is guided by fluoroscopy or non-fluoroscopic electro-anatomic mapping systems (EAMS). Either classic point-to-point radio-frequency (RF) catheter ablation or so-called single-shot-devices like cryo-balloons are used to achieve electrical isolation of the pulmonary veins and the left atrium (LA). Fluoroscopy-based systems render overlay images from pre-operative 3-D data sets which are then merged with fluoroscopic imaging, thereby adding detailed 3-D information to conventional fluoroscopy. EAMS provide tracking and visualization of RF catheters by means of electro-magnetic tracking. Unfortunately, current navigation systems, fluoroscopy-based or EAMS, do not provide tools to localize and visualize single-shot-devices like cryo-balloon catheters in 3-D. We present a prototype software for fluoroscopy-guided ablation procedures that is capable of superimposing 3-D datasets as well as reconstructing cyro-balloon catheters in 3-D. The 3-D cyro-balloon reconstruction was evaluated on 9 clinical data sets, yielding a reprojected 2-D error of $1.72 \mathrm{~mm} \pm 1.02 \mathrm{~mm}$
\end{abstract}

Keywords: Ablation Procedures, Cardiac Procedures, Cryo-Balloon, Electrophysiology, Localization, Reconstruction

\section{INTRODUCTION}

Atrial Fibrillation (AF), affecting approximately $2 \%$ of the population, is the most common arrhythmia and a leading cause of stroke [1,2]. The current standard in interventional treatment of AF is the Isolation of the Pulmonary Veins (PVI) [3]. By use of radiofrequency (RF) or cryo catheters, arrhythmogenic substrate located in the pulmonary veins (PVs) is electrically isolated from the left atrium (LA) [4]. PVI is performed under monoplane or biplane fluoroscopic guidance, additionally assisted by non-fluoroscopic electroanatomic mapping systems (EAMS) [5]. EAMS provide visualization and tracking of standard RF catheters, whereas cryo-balloon ablation catheters are not supported. Preprocedural cross-sectional imaging (MRI, CT) or rotational angiography is performed to evaluate LA and PV anatomy. 
Augmented fluoroscopy, using a perspectively forward projected overlay representation of 3-D objects onto live fluoroscopic images, has become a useful tool for navigation when performing ablation procedures [4-9]. A new software (Siemens AG, Forchheim, Germany) facilitates the superimposition of MRI, CT and C-arm CT data sets onto biplane fluoroscopy. Catheters, visualized in fluoroscopy, are captured by the software, reconstructed and integrated into the preprocedurally acquired 3D-dataset. Thereby the software provides guidance not only for RF but also for cryo-ballon catheter ablation. In our approach, the cryo-balloon catheter is modeled as a sphere in 3-D. Methods to reconstruct ellipsoids either require three views [10] or additional 3-D information [11]. A method for sphere reconstruction has already been proposed in [12], but it turns out to be rather sensitive to noise [13]. The method presented by Kleinoeder et al. [13] to reconstruct a cryo-balloon catheter from two views was proven to be more robust. It is well suited to compute a 3-D model even in the presence of noise. Used as part of a fluoroscopic overlay image for augmented fluoroscopy applications, the reprojection of a reconstructed cyro-balloon was evaluated on 9 data sets.

\section{SPHERE RECONSTRUCTION}

The reconstruction process consists of two steps [12]. In the first step, the 2-D input data is processed and in the second step, the 3-D catheter model is generated. As input, manually selected 2-D points on the boundary of the balloon-catheter in both views are needed, see Figure 1 for an example of a single view. These points are denoted as $\mathbf{p}_{A, i}, \mathbf{p}_{B, j} \in \mathbb{R}^{2}$ with $i, j \in \mathbb{N}$ for imaging plane $A$ and imaging plane $B$. The points hold image coordinates of plane $A \mathbf{p}_{A, i}=(u, v)^{T}$ and plane $B \mathbf{p}_{B, j}=(u, v)^{T}$ with $u, v, \in \mathbb{R}$. Two-dimensional ellipses are fitted to these points according to [14]. The ellipse parameters are given in matrix notation as $\mathbf{C}_{A}, \mathbf{C}_{B} \in \mathbb{R}^{3 \times 3}$. The 2-D points have to fulfill the following equations

$$
\begin{aligned}
\tilde{\mathbf{p}}_{A, i}^{T} \mathbf{C}_{A} \tilde{\mathbf{p}}_{A, i} & =0 \\
\tilde{\mathbf{p}}_{B, j}^{T} \mathbf{C}_{B} \tilde{\mathbf{p}}_{B, j} & =0
\end{aligned}
$$

with $\tilde{\mathbf{p}}_{A, i}=\left(\mathbf{p}_{A, i}, 1\right)^{T}$ and $\tilde{\mathbf{p}}_{B, j}=\left(\mathbf{p}_{B, j}, 1\right)^{T}$. Using these implicit ellipse parameters, 3-D cones $\mathbf{Q}_{A}, \mathbf{Q}_{B} \in \mathbb{R}^{4 \times 4}$ can be calculated by incorporating the projection matrices $\mathbf{P}_{A}, \mathbf{P}_{B} \in$ $\mathbb{R}^{3 \times 4}[15-18]$

$$
\begin{aligned}
\mathbf{Q}_{A} & =\mathbf{P}_{A}^{T} \mathbf{C}_{A} \mathbf{P}_{A} \\
\mathbf{Q}_{B} & =\mathbf{P}_{B}^{T} \mathbf{C}_{B} \mathbf{P}_{B} .
\end{aligned}
$$

For simplicity, we restrict ourselves to the calculation for plane $A$ only. The calculation for plane $B$ is analogous. The 3 -D cones mentioned above have the following structure

$$
\mathbf{Q}_{A}=\left(\begin{array}{cc}
\tilde{\mathbf{Q}}_{A} & \mathbf{a}_{A} \\
\mathbf{a}_{A}^{T} & \mu_{A}
\end{array}\right)
$$

with the left upper diagonal sub-matrix $\tilde{\mathbf{Q}}_{A} \in \mathbb{R}^{3 \times 3}$, the vector $\mathbf{a}_{A} \in \mathbb{R}^{3}$ and the scalar value $\mu_{A} \in \mathbb{R}[12]$. To align an arbitrary cone with the coordinate system, we have to find a transformation

$$
\mathbf{T}_{A}=\left(\begin{array}{cc}
\mathbf{R}_{A} & \mathbf{t}_{A} \\
0 & 1
\end{array}\right)
$$

with the rotation $\mathbf{R}_{A} \in \mathbb{R}^{3 \times 3}$ and the translation $\mathbf{t}_{A} \in \mathbb{R}^{3}$. The transformed cone $\hat{\mathbf{Q}}_{A} \in \mathbb{R}^{4 \times 4}$ is then given by [12]

$$
\hat{\mathbf{Q}}_{A}=\mathbf{T}_{A}^{T} \mathbf{Q}_{A} \mathbf{T}_{A} .
$$

Considering the left upper $3 \times 3$ sub-matrix of $\hat{\mathbf{Q}}_{A}$

$$
\mathbf{R}_{A}^{T} \tilde{\mathbf{Q}}_{A} \mathbf{R}_{A}
$$




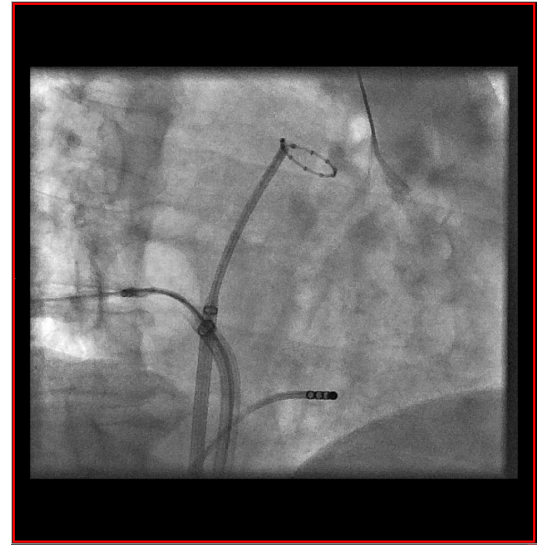

(a)

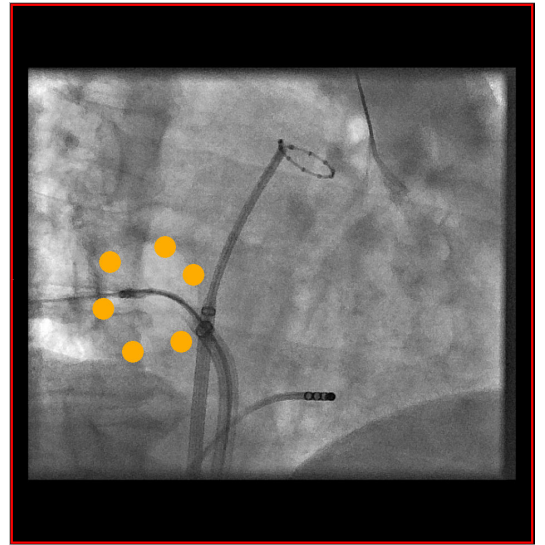

(b)

Figure 1: (a) The original fluoroscopic image during a regular atrial fibrillation procedure using a cryoballoon catheter. (b) The manually selected points of the balloon-catheter are superimposed onto the live fluoroscopic image (orange).

the rotation matrix $\mathbf{R}_{A}$ is given by the matrix of eigenvectors of $\tilde{\mathbf{Q}}_{A}$. The translation can then be computed by

$$
\mathbf{t}_{A}=-\tilde{\mathbf{Q}}_{A}^{-1} \mathbf{a}_{A}
$$

Given the rotation and translation, the central rays $\mathbf{r}_{A}, \mathbf{r}_{B} \in \mathbb{R}^{3}$ for both cones can be calculated and intersected by the method presented in [15] to find the center of the reconstructed sphere $\mathbf{w}_{c} \in \mathbb{R}^{3}$. To calculate the radius of the reconstructed sphere, each cone is intersected with a 3 -D plane parallel to the imaging plane at the position of the calculated center $\mathbf{w}_{c}$. This method is part of the 3-D ellipse reconstruction method presented in $[17,18]$. The intersection of a 3-D cone with a 3-D plane yields an ellipse in 3-D, especially in our case. The 3-D points of the ellipse lie on the sphere. Each point is now used to calculate the radius as described in [12]. The mean of all calculated radii yields the spheres' radius resulting from one cone. Averaging the radius results for both cones results the spheres' final radius.

\section{EVALUATION AND RESULTS}

For evaluation of our method, 9 clinical biplane sequences from 3 different patients were available. Our data was acquired on an Artis zee biplane C-arm X-ray system (Siemens AG, Healthcare Sector, Forchheim, Germany). The study was approved by the institutional Ethics Committee and the Bavarian State Board of physicians. Patients gave informed consent to the procedure and analysis of clinical data. The cryo-balloon catheters were manually outlined in the 2-D images. We posed no restriction upon the user, i.e., the cyro-balloons were selected as they were visible. Even though our reconstruction method assumes a circle in 2-D, the 2-D input was not restricted to be circular shaped. In most cases, the cryo-balloon is visible as an elliptical shaped structure. To calculate the error of our reconstruction method, we computed the 2-D distance between the manually selected points and the forward projected 3-D sphere into the imaging planes. Our method yielded a 2-D error of $1.72 \mathrm{~mm} \pm 1.02 \mathrm{~mm}$. The results are shown in Figure 2.

\section{DISCUSSION AND CONCLUSIONS}

During the course of this work, we found our sphere reconstruction method from two views robust and easy to use. The 2-D errors are, at least to a certain amount, due to the fact, that the cyro-balloon is not a perfect sphere. Our reconstruction method is designed to reconstruct a 


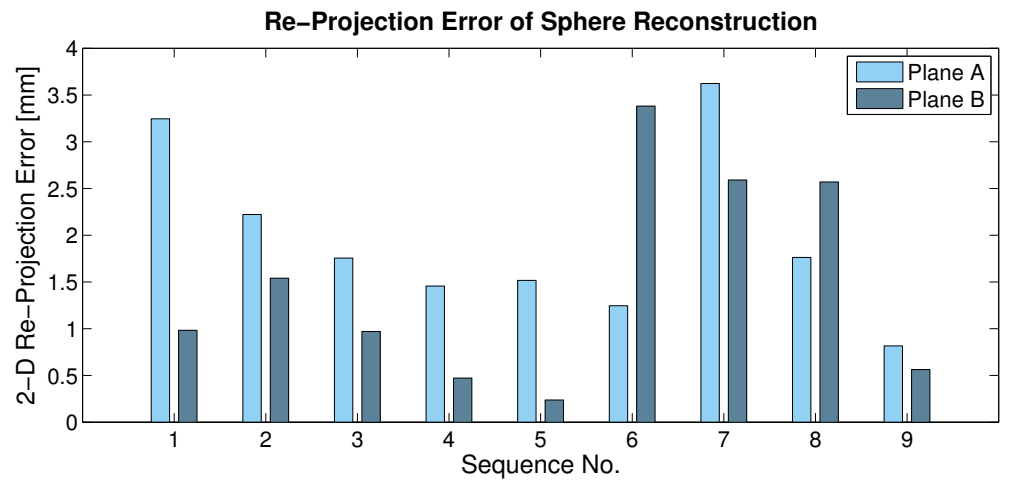

Figure 2: Projection of the reconstructed sphere yielded an average 2-D reconstruction error of $1.72 \mathrm{~mm} \pm 1.02 \mathrm{~mm}$.

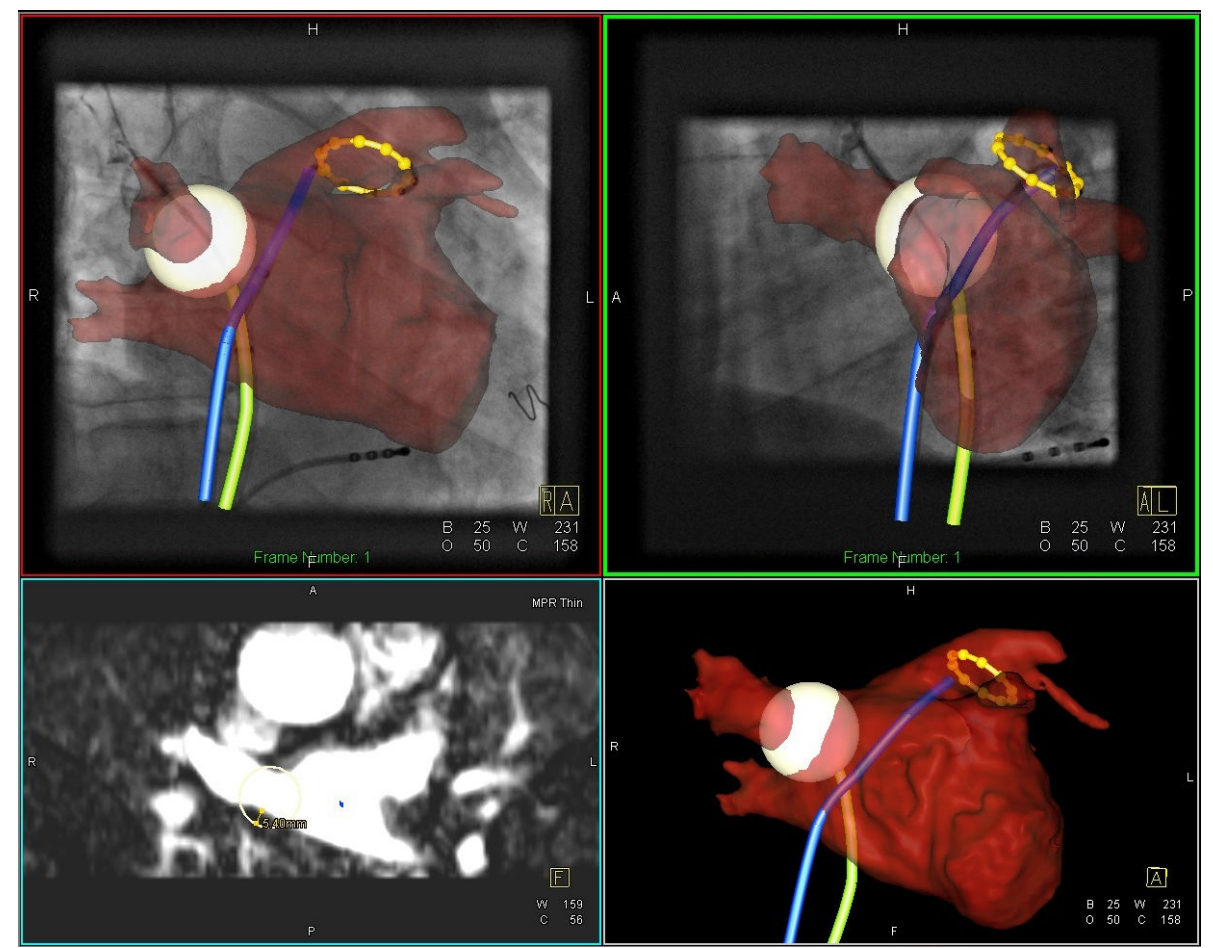

Figure 3: Upper left and right section: $-30^{\circ} / 60^{\circ}$ biplane fluoroscopic views, superimposed MRI derived LA-model, reconstructed $28 \mathrm{~mm}$ Cryo Balloon at right superior PV and reconstructed circular mapping catheter at left superior PV. Lower left section: Transversal MRI sectional view with visualized Balloon. Lower right section: Anterior view on LA-model including reconstructed catheters.

sphere, whereas in some cases, an ellipsoid in 3-D would be better. Unfortunately, an ellipsoid reconstruction from two views is ambiguous. Nevertheless, the proposed method is likely to be helpful during cryo-balloon catheter ablation procedures as it provides visual feedback to the physician, see Fig. 4 for an example. The overlay together with a segmented left atrium is shown in Fig. 3. In addition to that, previous balloon positions could be stored, in particular when multiple freezing treatments are applied to the same pulmonary vein, to improve the guidance and efficiency of the procedure. Using the 3-D visualization of our method, the physician could visually assess the fit of the cryo-balloon to the pulmonary vein, see Fig. 5. We expect that the use of our approach will further improve the safety and efficacy of this treatment option. 


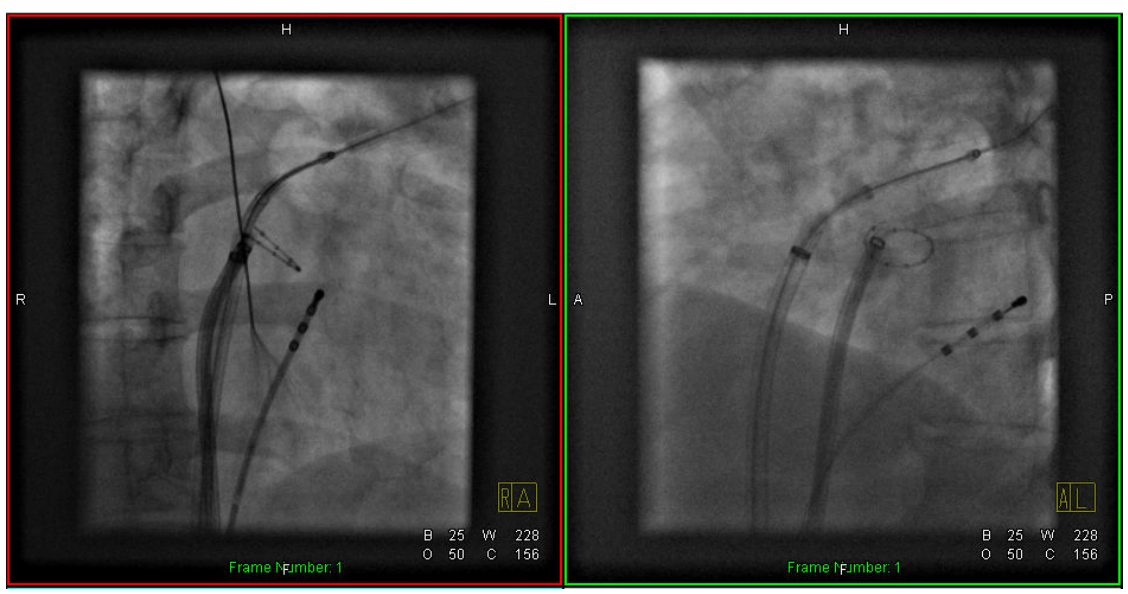

(a)

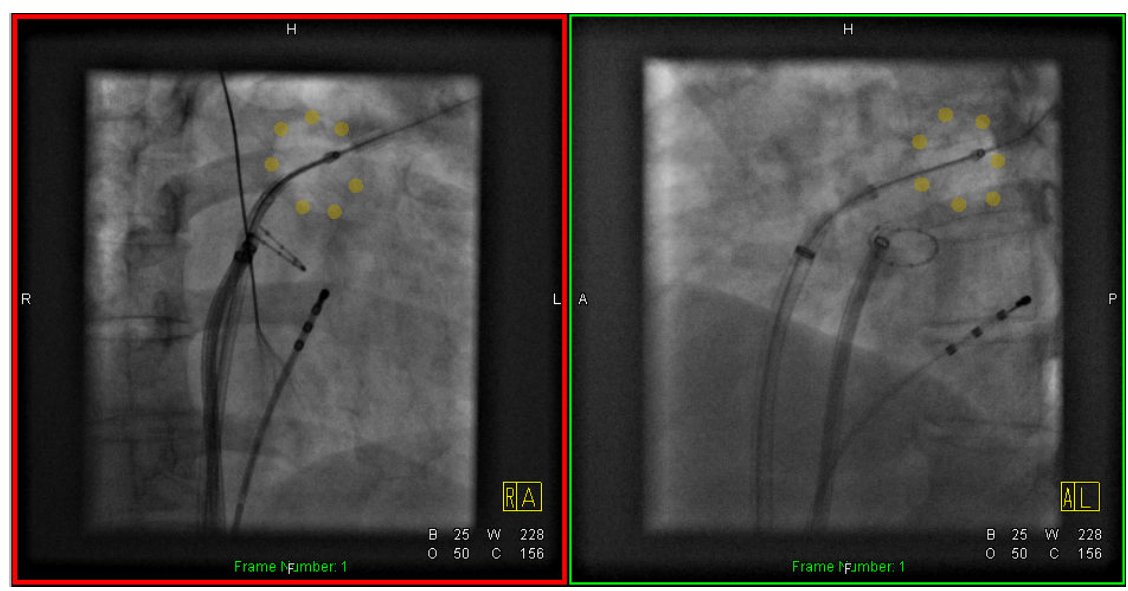

(b)

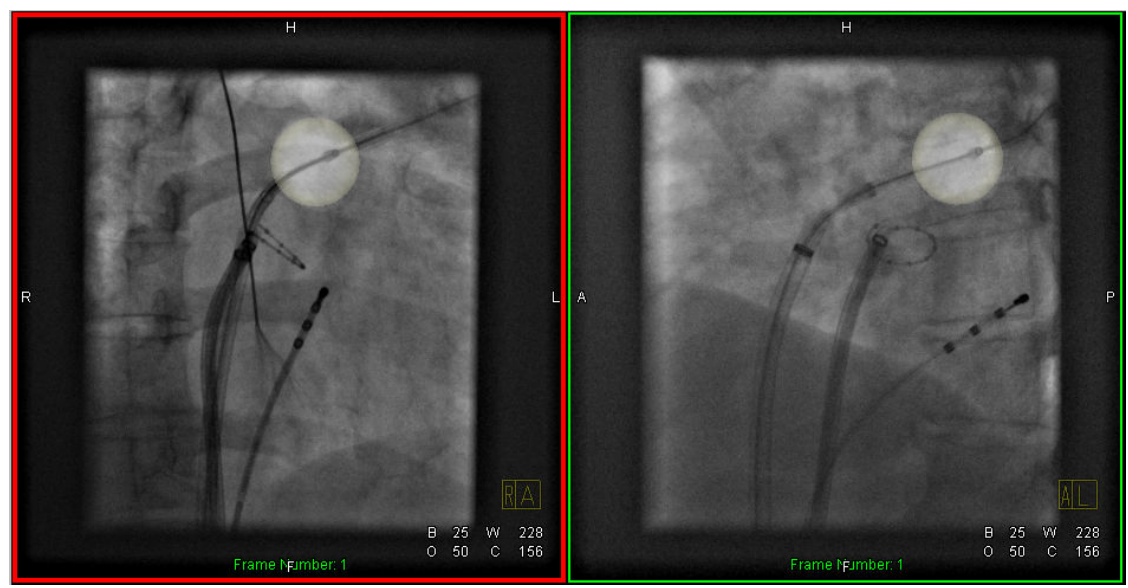

(c)

Figure 4: (a) Biplane fluoroscopic sequence. (b) The same sequence with 2-D input points. (c) Reconstructed 3-D cyro-balloon overlayed onto fluoroscopic images. 


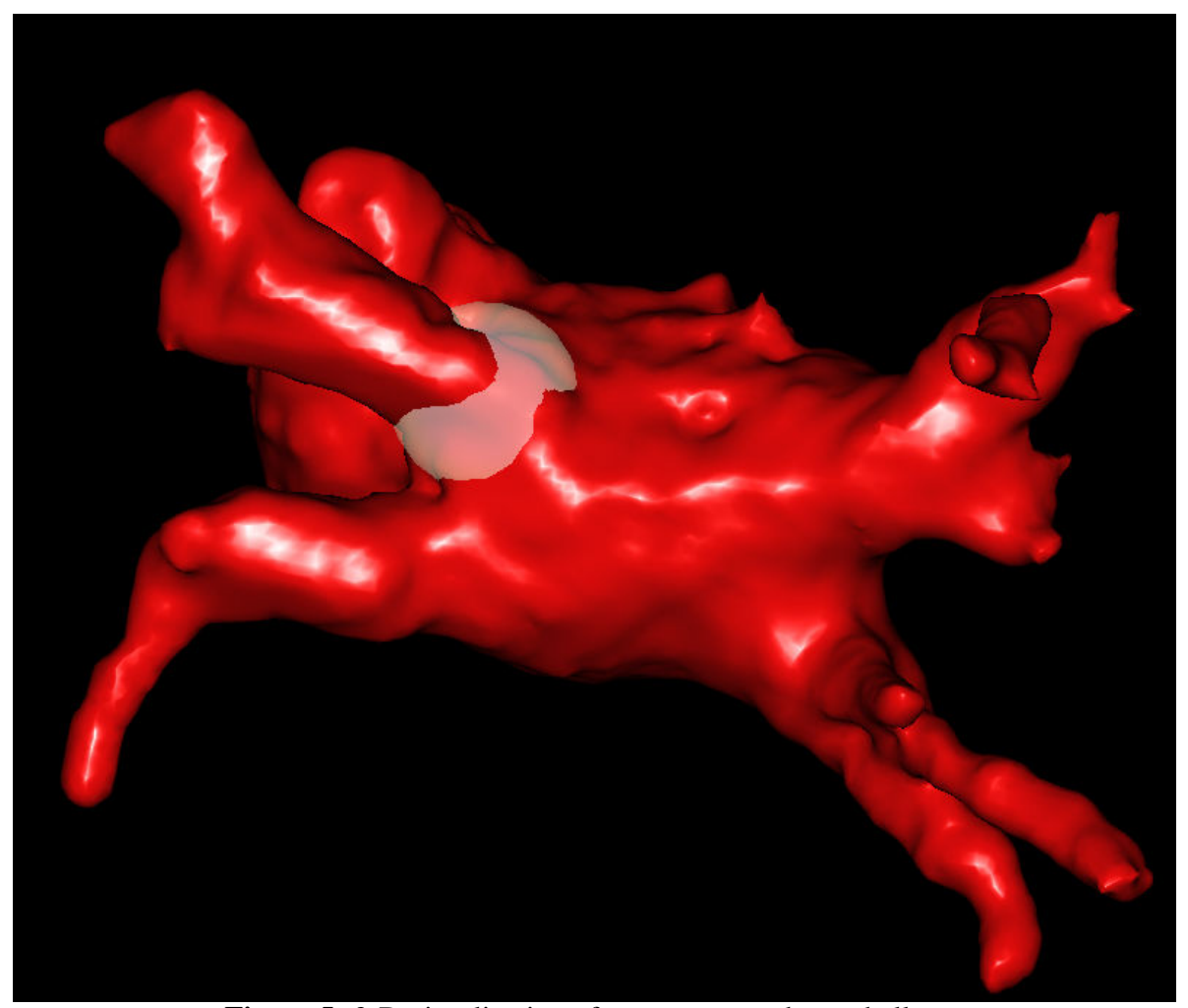

Figure 5: 3-D visualization of a reconstructed cryo-balloon.

Superimposition of MRI-datasets on biplane fluoroscopic imaging and sphere reconstruction is helpful to guide cryo PVI. In contrast to EAMS, the developed software supports navigation and documentation of cryo-balloon ablation procedures. Compared to conventional fluoroscopy, augmented fluoroscopy can improve orientation and catheter navigation in cryo PVI procedures. This novel imaging technology offers the capability to reduce fluoroscopy time, as reported by studies using monoplane CT-derived fluoroscopy overlay techniques [6]. Whereas current commercially available 3-D overlay systems may only process CT datasets, this new software features the handling of MRI datasets, too. The possibility of using MRI instead of CT for LA visualization additionally decreases radiation exposure for the patient.

\section{Acknowledgments}

This work has been supported by the German Federal Ministry of Education and Research $(\mathrm{BMBF})$ in the context of the initiative Spitzencluster Medical Valley - Europäische Metropolregion Nürnberg, project grant Nos. 01EX1012A and 01EX1012E, respectively. Additional funding was provided by Siemens AG, Healthcare Sector.

\section{References}

[1] M. Haïssaguerre, P. Jaïs, D. C. Shah, A. Takahashi, M. Hocini, G. Quiniou, S. Garrigue, A. Le Mouroux, P. Le Métayer, and J. Clémenty, "Spontaneous initiation of atrial fibrillation by ectopic beats originating in the pulmonary veins," New England Journal of Medicine 339(10), 659-666 (1998).

[2] S.-A. Chen, M.-H. Hsieh, C.-T. Tai, C.-F. Tsai, V. S. Prakash, W.-C. Yu, T.-L. Hsu, Y.-A. Ding, and M.-S. Chang, "Initiation of atrial fibrillation by ectopic beats originating from the pulmonary veins : Electrophysiological characteristics, pharmacological responses, and effects of radiofrequency ablation," Circulation 100(18), 1879-1886 (1999). 
[3] A. J. Camm, P. Kirchhof, G. Y. Lip, U. Schotten, I. Savelieva, S. Ernst, I. C. V. Gelder, N. Al-Attar, G. Hindricks, B. Prendergast, H. Heidbuchel, O. Alfieri, A. Angelini, D. Atar, P. Colonna, R. D. Caterina, J. D. Sutter, A. Goette, B. Gorenek, M. Heldal, S. H. Hohloser, P. Kolh, J.-Y. L. Heuzey, P. Ponikowski, and F. H. Rutten, "Guidelines for the management of atrial fibrillation,” European Heart Journal 31, 2369-2429 (2010).

[4] T. Arentz, R. Weber, G. Bürkle, C. Herrera, T. Blum, J. Stockinger, J. Minners, F. J. Neumann, and D. Kalusche, "Small or large isolation areas around the pulmonary veins for the treatment of atrial fibrillation?," Circulation 115(24), 3057-3063 (2007).

[5] M. J. Earley, R. Showkathali, M. Alzetani, P. M. Kistler, D. Gupta, D. J. Abrams, J. A. Horrocks, S. J. Harris, S. C. Sporton, and R. J. Schilling, "Radiofrequency ablation of arrhythmias guided by non-fluoroscopic catheter location: a prospective randomized trial," European Heart Journal 27(10), 1223-1229 (2006).

[6] S. Knecht, H. Skali, M. D. O’Neill, M. Wright, S. Matsuo, G. M. Chaudhry, C. I. Haffajee, I. Nault, G. H. M. Gijsbers, F. Sacher, F. Laurent, M. Montaudon, O. Corneloup, M. Hocini, M. Haissaguerre, M. V. Orlov, and P. Jais, "Computed tomography-fluoroscopy overlay evaluation during catheter ablation of left atrial arrhythmia," Europace 10, 931938 (2008).

[7] De Buck, S., Maes, F., Ector, J., Bogaert, J., Dymarkowski, S., et al., "An augmented reality system for patient-specific guidance of cardiac catheter ablation procedures," IEEE Trans. Med. Imaging 24(11), 1512-1524 (2005).

[8] J. Ector, S. De Buck, et al., "Biplane three-dimensional augmented fluoroscopy as single navigation tool for ablation of atrial fibrillation: Accuracy and clinical value," Heart Rhythm 5, 957-964 (2008).

[9] Ector, J., Buck, S.D., Loeckx, D., Coudyzer, W., et al., "Changes in Left Atrial Anatomy Due to Respiration: Impact on Three-Dimensional Image Integration During Atrial Fibrillation Ablation," J Cardiovasc. Electr. 19(7), 828-834 (2008).

[10] S. Ma and L. Li, "Ellipsoid Reconstruction from Three Perspective Views," Pattern Recognition, International Conference on 1, 344 (1996).

[11] S. Wijewickrema, A. Paplinski, and C. Esson, "Reconstruction of ellipsoids on rollers from stereo images using occluding contours," in International Conference on Computer Vision Theory and Applications, (Setubal, Portugal) (2006).

[12] S. Wijewickrema, A. Paplinski, and C. Esson, "Reconstruction of Spheres using Occluding Contours from Stereo Images," in Proceedings of the 18th International Conference on Pattern Recognition (ICPR'06), (2006).

[13] A. Kleinoeder, A. Brost, F. Bourier, M. Koch, K. Kurzidim, J. Hornegger, and N. Strobel, "Cryo-Balloon Reconstruction from Two Views," in Proceedings of IEEE International Conference on Image Processing, IEEE, Ed., 989-992 (2011).

[14] R. Halir and J. Flusser, "Numerically Stable Direct Least Squares Fitting Of Ellipses," in WSCG (International Conferences in Central Europe on Computer Graphics, Visualization and Computer Vision), February 9 - 13, 1998, University of West Bohemia, Campus Bory, Plzen - Bory, Czech Republic, 253-257 (1998).

[15] A. Brost, N. Strobel, L. Yatziv, W. Gilson, B. Meyer, J. Hornegger, J. Lewin, and F. Wacker, "Accuracy of x-ray image-based 3D localization from two C-arm views: a comparison between an ideal system and a real device," in Medical Imaging 2009: Visualization, Image-Guided Procedures, and Modeling, M. Miga and K. Wong, Eds., 7261, 72611Z, SPIE, (Lake Buena Vista, FL, USA) (2009).

[16] A. Brost, N. Strobel, L. Yatziv, W. Gilson, B. Meyer, J. Hornegger, J. Lewin, and F. Wacker, "Geometric Accuracy of 3-D X-Ray Image-Based Localization from Two CArm Views," in Workshop on Geometric Accuracy In Image Guided Interventions - Medical Image Computing and Computer Assisted Interventions 2009, September, 20, London, UK, 12-19, MICCAI, (London UK) (2009). 
[17] A. Brost, R. Liao, J. Hornegger, and N. Strobel, “3-D Respiratory Motion Compensation during EP Procedures by Image-Based 3-D Lasso Catheter Model Generation and Tracking," in MICCAI 2009, September 20 - 24, London, UK, LNCS 5761, 394-401, Springer Berlin / Heidelberg (2009).

[18] A. Brost, R. Liao, N. Strobel, and J. Hornegger, "Respiratory motion compensation by model-based catheter tracking during EP procedures," Medical Image Analysis 14(5), 695 - 706 (2010). Special Issue on the 12th International Conference on Medical Image Computing and Computer-Assisted Intervention (MICCAI) 2009. 